Introduction Presenteesim, as well as medical/pharmaceutical costs, have attracted attention in the occupational health field in Japan. Presenteeism and health risks are known to be associated. However, the relationship between health risks and total burden of presenteeism, absenteeism, and medical/pharmaceutical cost have only received scarce attention. We aimed to investigate this relationship by examining the data of Japanese workers.

Methods We conducted this study using the data of $>40$ yearold workers obtained from four pharmaceutical companies and health insurance associations. The workers were classified into three categories (low-risk, middle-risk, and high-risk) according to probabilities of developing cardiovascular disease, as calculated by risk factors (e.g., smoking, alcohol intake, and Body Mass Index) obtained from a previous study. Health data from self-administered questionnaires completed by the workers in 2014 were collected to evaluate presenteeism, and from compensation claims from the health insurance associations to calculate medical/drug costs. To measure presenteeism, we used the QQ method. We received medical (in-/out-patient) and drug (out-patient) data of all study samples from April 2014 to March 2015.

Results The total sample in the final analysis numbered 7808 (low-risk: 2755, middle-risk: 2672, and high-risk: 2381). The total cost for the high-risk group was the highest of all three groups ( $€ 4,138 /$ year/person), followed by the middle-risk group (€4,068/year/person), then the low-risk group $(€ 3,620 /$ year/person). Presenteeism was the largest cost in all groups, with the cost being the highest for the middle-risk group among all three groups.

Discussion Many interventions have been conducted for highrisk workers in Japan, but ones for low- and middle-risk workers remain insufficient. Our findings suggest that greater awareness is needed about the intervention needs of low- and middle-risk workers, especially with regard to presenteeism. To increase this awareness, we plan to proceed with further study and make a detailed proposal.

\section{MEASURING PRECARIOUS EMPLOYMENT IN EUROPE EIGHT YEARS INTO THE GLOBAL CRISIS}

${ }^{1}$ Nuria Matilla-Santander*, ${ }^{1}$ Cristina Lidón-Moyano, ${ }^{1}$ Adrián González-Marrón, ${ }^{1}$ Kailey Bunch, 'Juan Carlos Martín-Sánchez, 1,2Jose M Martínez-Sánchez. 'Group of Evaluation of Health Determinants and Health Policies, Sant Cugat del Vallès, Spain; ${ }^{2}$ Tobacco Control Unit, Hospitalet de Llobregat, Spain

\subsection{6/oemed-2018-ICOHabstracts.378}

Introduction Precarious employment is considered an emerging social determinant of health; its prevalence is increasing and its study remains in its infancy. The objective of this study is to describe the prevalence of precarious employment in the European Union (EU-28) using a multidimensional approach, 8 years into the economic crisis.

Methods This is a cross-sectional study based on data from the Flash Eurobarometer 398 among salaried workers of the EU-28 from $2014(n=7702)$. We derived 4 factors of precarious employment (not having the ability to exercise rights, vulnerability, disempowerment and temporariness) from several items of the questionnaire. We calculated the proportions and their $95 \%$ confidence intervals $(95 \% \mathrm{CI})$ for each of the precarious employment factors. Also, we calculated the prevalence of precarious employment (having at least one factor) and the proportion of workers presenting 1, 2, 3 or 4 factors of precarious employment. All analyses were stratified by age, sex, age at the end of education and welfare regime.

Results $66.38 \%$ (95\% CI: 60.58 to 71.72 ) of the salaried European workers had precarious employment. The prevalence of precarious employment was higher in Eastern European countries (72.64\%; 95\% CI: 61.78 to 81.34) than Nordic European countries (51.09\%; 95\% CI: 44.38 to 57.77$)$. No differences were found according to sex, age, or age at the end of education. The most prevalent factor was not having the ability to exercise rights (42.40\%), followed by disempowerment (31.44\%), vulnerability (12.41\%) and temporariness (11.36\%).

Conclusion 2 out of 3 European salaried workers suffer precarious employment. Precariousness is widespread among the salaried working population as shown by the similar prevalence found between men and women, workers of different ages and age at the end of education. The European Commission should consider new forms of employment and legislate accordingly to avoid an increase in precarious employment.

\section{THE EFFECT OF SHIFT WORK ON CARDIOMETABOLIC HEALTH: FINDINGS FROM THE ATLANTIC PATH COHORT STUDY}

${ }^{1}$ Ellen Sweeney, ${ }^{1} Z$ hijie Michael Yu, ${ }^{2}$ Trevor JB Dummer, ${ }^{1}$ Yunsong Cui, ${ }^{1}$ Vanessa DeClercq, ${ }^{1}$ Cynthia Forbes, ${ }^{3}$ Scott A Grandy, ${ }^{3}$ Melanie Keats, ${ }^{4}$ Louise Parker, ${ }^{5}$ Anil Adisesh*. ${ }^{1}$ Atlantic PATH, Population Cancer Research Program, Dalhousie University, Halifax, Nova Scotia, Canada; ${ }^{2}$ Centre of Excellence in Cancer Prevention, School of Population and Public Health, University of British Columbia, Vancouver, Canada; ${ }^{3}$ School of Health and Human Performance, Dalhousie University, Halifax, Nova Scotia, Canada; ${ }^{4}$ Department of Paediatrics, Dalhousie University, Halifax, Nova Scotia, Canada; ${ }^{5}$ Dalhousie Medicine New Brunswick, Dalhousie University, Saint John, New Brunswick, Canada

\subsection{6/oemed-2018-ICOHabstracts.379}

Introduction Contemporary work environments increasingly rely upon a 24 hour work cycle resulting in more employees exposed to shift work. $30 \%$ of working age Canadians work evening, night and rotating shifts, and $21 \%$ of workers in the European Union. Compared to regular daytime work, shift work has the potential for disturbing sleep patterns and disrupting circadian rhythms with adverse health effects.

Methods Participation was limited to volunteers from the Atlantic Canadian Provinces (Nova Scotia, New Brunswick, Newfoundland and Labrador, and Prince Edward Island). 12413 participants, including 4155 shift workers and 8258 non-shift workers (matched 1:2 by age, sex, and education) from the Atlantic Partnership for Tomorrow's Health (PATH) study. Multiple general linear and logistic regression models were used to assess differences in body adiposity and self-reported cardiometabolic disease outcomes between shift workers and non-shift workers.

Results There was a significant increased risk of obesity and diabetes among shift workers compared to their matched controls. Shift workers were $18 \%$ more likely to be obese (95\% CI: 9 to 29 ) and $8 \%$ more likely to have abdominal obesity (95\% CI: 0 to 17). Shift workers were $31 \%$ more likely to have diabetes than non-shift workers (95\% CI: 11 to 56). The strength of this association was further demonstrated by controlling for participants' fat mass index (FMI), which resulted in a $28 \%$ increased risk of diabetes among shift workers (95\% CI: 2 to 60). Despite the increased likelihood of being physically active, regular night shift workers had higher levels of BMI, waist circumference, and fat mass compared with matched controls. 
Conclusion Despite higher levels of physical activity and lower levels of sedentary behaviour, shift workers were more likely to have increased rates of diabetes and obesity and are subsequently at increased the risk of developing other chronic disease. The effects of shift work on cardiometabolic status may be independent of simple obesity.

\section{COLLECTING AND ORGANISING BASIC OCCUPATIONAL HEALTH DATA FOR INTERNATIONAL COMPARISONS}

${ }^{1} \mathrm{~A}$ Tsutsumi* ${ }^{2} \mathrm{~S}$ Kajiki, ${ }^{3} \mathrm{~T}$ Muto, ${ }^{1} \mathrm{~A}$ Shimazu, ${ }^{2} \mathrm{~S}$ Okahara, ${ }^{4} \mathrm{~K}$ Ohdo, ${ }^{4} \mathrm{~T}$ Yoshikawa, ${ }^{5} \mathrm{~T}$ Mishiba, ${ }^{1} \mathrm{~A}$ Inoue. ${ }^{1}$ Kitasato University, Kanagawa, Japan; ${ }^{2}$ University of Occupational and Environmental Health, Fukuoka, Japan; ${ }^{3}$ Dokkyo Medical University, Tochigi, Japan; ${ }^{4}$ National Institute of Occupational Safety and Health, Tokyo, Japan; ${ }^{5}$ Kindai University, Osaka, Japan

\subsection{6/oemed-2018-ICOHabstracts.380}

Introduction In regard to international research on occupational health, field survey reports concerning occupational health institutions and related professionals overseas have increased, resulting in an increased number of international comparative studies. However, obtaining mortality and sick leave statistics as well as basic data on industrial accidents overseas still remain difficult because these data have never been collected or, if they have been collected, have never been organised. A research group has been launched to collect basic data, including the above-mentioned data, of as many countries as possible as well as organising the data. The aim of the study is to obtain knowledge that will develop into international comparative studies.

Methods The steps we are taking consist of

i. collecting new data and information that the ILO, WHO and other international organisations and Japanese research institutes possess or have published,

ii. combining these with the data and information held by Japanese occupational health researchers as well as the results of bibliographic searches, and

iii. comparing these with the data of other countries and showing them in an organised form.

Results We have made the lists to be collected as follows and started to collect the data with the worldwide base:

a. Occupational disease statistics (by industry, disease and year)

b. Mortality statistics due to industrial accidents

c. Off-the-job injury and sickness statistics (with necessary definitions)

d. Implementation status of health examinations and special health examinations (if actually performed), together with examination items and the rate of abnormal findings

e. Actual benefit payment under industrial accident compensation insurance (if there is an insurance plan or similar system)

f. Suicide statistics

g. Actual management of chemical substances

h. Other necessary points in your survey of worker health in your country

i. Have any industrial accident prevention plans or similar programs been established by the government? If so, what are the rates of accomplishment and achievement of the plans?

j. Statistics related to the basic work force of your country.
The preliminary findings include that there are no systematic registries on some occupational diseases statistics even in industrialised countries, and for international comparison it is necessary to get the information on how to collect statistics which may vary between countries.

Conclusion Our research will enable having basic knowledge that contributes to improving occupational health standards. Simultaneously, we intend to summarise our work, recommending the benefits of using these basic data, and indicating matters requiring further solutions and the prospects for international comparisons. The results will be published on our website for general use and in scientific journals.

Acknowledgement This research is supported by the Occupational Health Promotion Foundation of Japan.

\section{HEALTH BURDEN FROM INJURIES AMONG WORKING POPULATIONS IN THAILAND}

O Untimanon*, K Boonmeephong, T Saipang, K Sukanun, A Promrat. Bureau of Occupational and Environmental Diseases, Department of Disease Control, Ministry of Public Health, Thailand

\subsection{6/oemed-2018-ICOHabstracts.381}

Introduction Injury causes an enormous amount of physical, financial and emotional hardship for working populations, their families and workplaces. It has been identified as the top of occupational health problems in Thailand. This descriptive study aimed to investigate:

- the situation of data entry regarding to occupational injuries

- the unintentional injury rates among working populations with breakdown by gender, age and type of occupation, and

- the health burden from such injuries.

Methods Data were collected from thirty-one participated hospitals. Such data were manipulated and analysed using frequency, percentage and Disability-Adjusted Life Years (DALYs). A number of injured patients were adjusted with the proportion of those injured patients who did not access the services at general/regional hospitals.

Results The results showed that $38.71 \%$ of the hospitals did not notify ICD-10 coding for the occupational injuries into the hospital database. The injury rates were 102.43 per 1000 working populations and the injured fatality rates were 88.60 per 100000 working populations. Such rates were higher in males than females with age 35-44 years old. The burdens of unintentional injuries among 31 hospitals in males and females were 62.29 DALYs and 23.23 DALYs per 1000 working populations, respectively. Of DALYs, Year of Life Loss (YLL) was higher than Year Lost due to Disability (YLD).

Conclusion This study recommended that unintentional injuries prevention from occupational exposures should address on industrial work and agriculture work. Such results are useful to support 'safety Thailand' project which is aimed to integrate and promote collaboration on safety and occupational health among concerned ministries. According to the under-report of occupational injuries, it is needed to train the staff of the hospital to record ICD-10 code for occupational injuries in the database. Such data are crucial for the planning and guiding of preventive strategies for occupational health injurie. 